Check for updates

Cite this: RSC Adv., 2017, 7, 51711

\title{
Rapid and efficient intense pulsed light reduction of graphene oxide inks for flexible printed electronics
}

\author{
Limin Pei and Yu-Feng Li (D) *
}

In this study, a printable GO ink was prepared by dispersing $120 \mathrm{mg} \mathrm{GO}$ in $30 \mathrm{ml}$ mixed solvents (deionized water : ethanol : ethylene glycol $=1: 1: 1$ ). The prepared ink was inkjet-printed onto the flexible substrate PET. Printed patterns were reduced with intense pulsed light (IPL) without damaging the substrate PET. By adjusting the distance between the intense light and the patterns and number of pulses in a given time, patterns with low resistance and good flexibility were achieved. Upon printing 20 passes, the resistance decreased from 56.77 M $\Omega$ for the patterns before IPL treatment to $760.4 \Omega$ for the patterns after IPL treatment. It was lowered by nearly four orders of magnitude. It was proved that IPL reduction on the GO ink could be used as an effective method for the synthesis of flexible electronics such as sensors.

Received 20th September 2017 Accepted 25th October 2017

DOI: $10.1039 / c 7 r a 10416 b$

rsc.li/rsc-advances research on graphene oxide (GO) as an ink material has been extensively conducted. ${ }^{19-21}$ GO has functionalized oxygencontaining groups on edges and basal planes; this makes GO easy to suspend in water or other organic solvents without any additional stabilized polymers and remain stable for a long time. ${ }^{10,22}$ However, conductivity of GO is far lower than that of graphene, and it needs additional reduction processing, such as use of a reducing agent ${ }^{20}$ and annealing, ${ }^{23}$ to restore its structure and obtain high conductivity. ${ }^{19}$ In $2014, \mathrm{Chi}^{24}$ et al. synthesized a freestanding nanohybrid paper by inkjet printing, and the paper was reduced to a graphene paper-supported threedimensional porous graphene hydrogel-polyaniline nanocomposite in a HI solution; moreover, the flexible all-solid-state supercapacitor fabricated using this nanocomposite exhibited excellent flexibility and acceptable energy density. In 2015, Xiao ${ }^{25}$ et al. utilized the reducing agent $\mathrm{HI}$ solution to develop the RGO/ PANI/RGO sandwich-structured nanohybrid paper. It revealed high conductivity, lightweight, excellent mechanical property, and chemical stability, making it a potential material for supercapacitors and active electrodes. However, common reducing agents are poisonous, ${ }^{21}$ and annealing reduction is not suitable for temperature-sensitive substrates such as polyethylene terephthalate (PET), and polyimide (PI). ${ }^{20}$

IPL technology is carried out under low temperature, selective, and non-contact conditions. Using wideband spectrum and high energy pulsed light, ink materials on various substrates can be sintered and processed to realize functionality. ${ }^{26}$ In 2011, Kang ${ }^{27}$ et al. of the Hahn research group studied sintering of the silvernanoparticle inks by intense pulsed light at room temperature. By adjusting the powers and the numbers of pulses, the silver electrode was completely sintered, and a combination of well binding ability with a low resistivity of $50 \mathrm{n} \Omega \mathrm{m}$ was realized. In $2015, \operatorname{Secor}^{28}$ et al. sintered a graphene-based ink printed on the flexible substrate PET by intense pulsed light. They produced
School of Materials Science and Engineering, Shenzhen Graduate School, Harbin Institute of Technology, HIT Campus, Shenzhen University Town, Xili, Nanshan, Shenzhen 518055, PR China. E-mail: yfli@hit.edu.cn; Fax: +86-755-26033504; Tel: $+86-755-26038236$ 
inkjet-printable graphene-based inks with high concentrations of up to $20 \mathrm{mg} \mathrm{ml}^{-1}$ and realized excellent conductivity of $25000 \mathrm{~S} \mathrm{~m}^{-1}$ after one printing pass with pulsed light sintering. Thus, the IPL technology has great potential in printed electronics. However, research on the application of the IPL method for the reduction of GO ink or exploration of the effects of different processing parameters on the properties, such as the surface morphology and conductivity, of the treated patterns after reduction is rarely reported. To investigate the influence of IPL technology on GO inks, in this study, the IPL technology was used to reduce printed GO patterns on PET without damaging the substrate. The morphology and the conductivity of the printed patterns were characterized and compared, and the flexibility of the reduced patterns was explored.

\section{Experimental}

\subsection{Preparation of the GO ink}

GO inks were synthesized by sonicating GO (purchased from Suzhou Tanfeng Graphene Technology Co. Ltd., 99\% purity) in solvents for $2 \mathrm{~h}$. The solvents involved deionized water, ethylene glycol (purchased from Tianjin Baishi Chemical Co. Ltd., 99.7\%), and ethanol (purchased from Tianjin Baishi Chemical Co. Ltd., 99.7\%).

\subsection{Substrate treatment}

To enhance the wetting activities of the hydrophobic substrate PET (purchased from Dejin Plastic Insulation Materials Co. Ltd., $R_{\mathrm{a}} \approx 0.5 \mu \mathrm{m}$ ), oxygen-plasma treatment was carried out to improve the wetting and spreading behavior of inks on the substrate. The power was set as $45 \mathrm{~W}$, and the time was set as $1 \mathrm{~min}$. Before treatment, the substrate was cleaned with ethanol to remove pollution particles.

\subsection{Inkjet printing}

A standard commercial inkjet printer Dimatix DMP-2831 was used to print designed patterns at room temperature. Rinsed DMC-11610 cartridges were injected with prepared GO inks and placed still for few minutes before printing the designed patterns to equilibrate the GO inks in it. The patterns printed on the treated substrate PET were squares with $10 \mathrm{~mm}$ length and $4 \mathrm{~mm}$ width.

\subsection{Reduction of the printed patterns}

Printed patterns were put under pulsed light to achieve high conductivity. By changing different parameters, their effects on conductivity and morphology of the printed patterns were explored. The printed patterns were dried for further investigation.

\subsection{Characterization}

The surface tension of the prepared GO inks was measured by SAT-5100 from RHESCA. The viscosity of the GO inks was measured by a Brookfield DV2T viscometer. The contact angles of the GO inks on the treated and untreated substrate PET were surveyed by a SL150L optical contact angle \& interface tension meter obtained from USA KINO Industry Co. Ltd. Atomic force microscopy (AFM) measurements were used to observe the morphology using the Bruker Multimode 8 with a NanoScope $\mathrm{V}$ Controller. The morphologies of the patterns before and after reduction were observed by a Hitachi S-470 field-scanning electron microscope (SEM). Raman spectra of the patterns were obtained using the Renishaw Invia Reflex Raman microscope with a $531 \mathrm{~nm}$ laser. XPS spectra of the patterns were acquired using an ESCALAB 250Xi spectrometer obtained from Thermo Fisher. Moreover, four-point sheet resistances of the

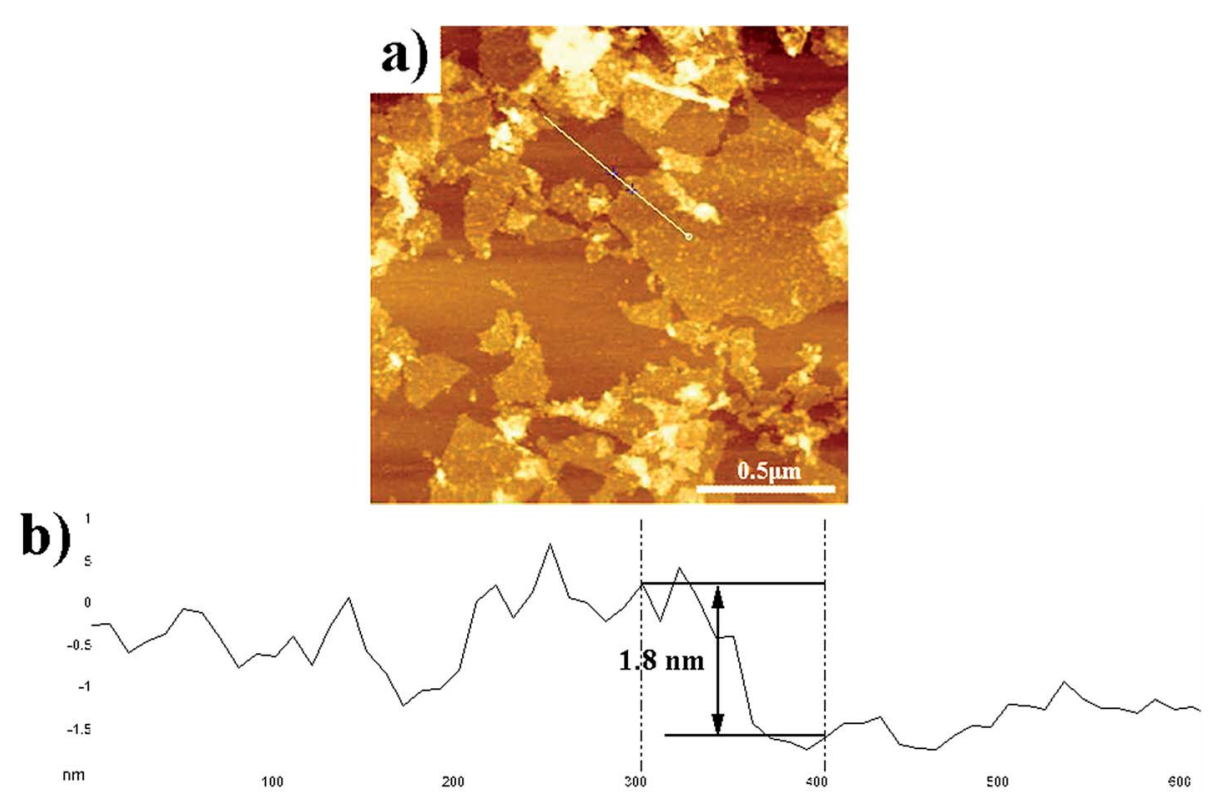

Fig. 1 AFM image of the GO sheets deposited on the mica substrates: (a) the surface morphology of GO sheets. (b) The height profile of GO sheets along the white line shown in (a). 
printed patterns were measured by a SB4120/2 test bench equipped with a Keithley multimeter.

\section{Results and discussion}

\subsection{Ink and substrate preparation}

Viscosity $(\gamma)(\mathrm{mPa} \mathrm{s})$, surface tension $(\eta)\left(\mathrm{mN} \mathrm{m}^{-1}\right)$, density $(\rho)$ $\left(\mathrm{g} \mathrm{cm}^{-3}\right)$, and the diameter of the printer nozzle $(a)(\mu \mathrm{m})$ have influences on the image quality and the printing process. ${ }^{15,29}$ Generally, the value of $Z$ calculated from $Z=[(a \rho \eta) / \gamma]^{1 / 2}$ must be between 1 and 14, and this can promise a stable and successful printing process. ${ }^{1}$ Diameter of the printer nozzle used was $22 \mu \mathrm{m}$. By adjusting the viscosity and the surface tension, a printable GO ink was finally prepared by dispersing $120 \mathrm{mg}$ GO sheets in $30 \mathrm{ml}$ solvents (deionized water : ethanol :ethylene glycol $=1: 1: 1$ ) and then sonicating the dispersion for $2 \mathrm{~h}$. It was estimated that $\gamma \approx 9.04 \mathrm{mPa} \mathrm{s}, \eta \approx 56.315 \mathrm{~m}$ $\mathrm{Nm}^{-1}, \rho \approx 0.9972 \mathrm{~g} \mathrm{~cm}^{-3}$, and this provided $Z \approx 3.89$, within the conventional range. It was seen that the ink was dark brown, lateral dimension of GO sheets in the ink was almost smaller a)

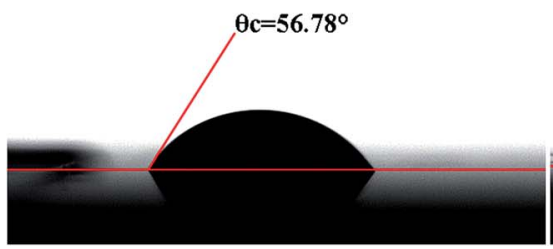

b)

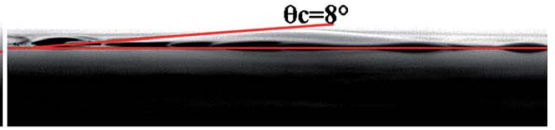

Fig. 2 The contact angle of the GO ink on different substrates: (a) untreated PET and (b) treated PET.

\section{a)}
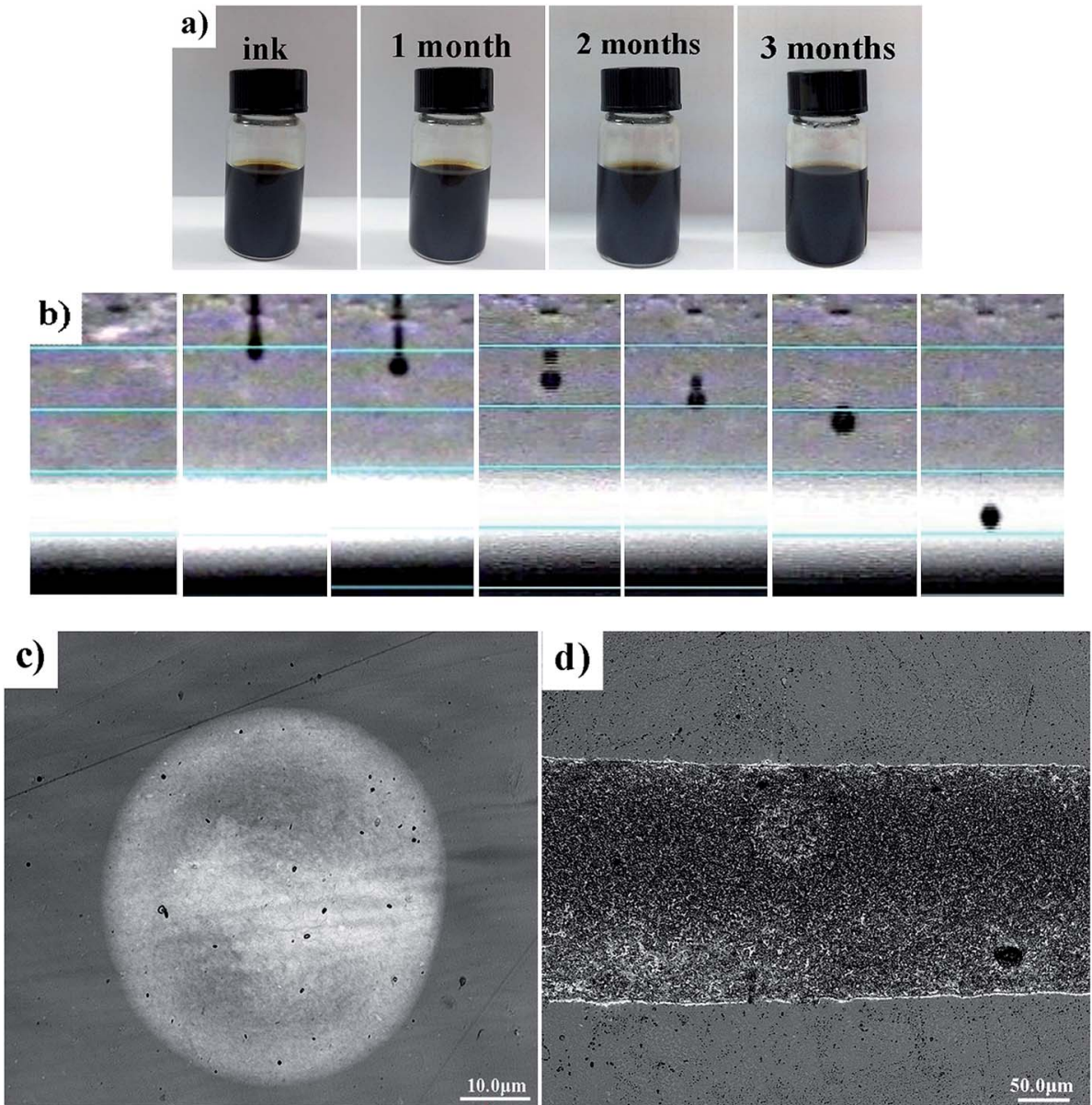

Fig. 3 Schematic of ink and printing: (a) stability of the prepared ink changing with time (just-prepared; one month; two months; and three months); (b) the injecting procedure of the ink from nozzles; (c) the morphology of a printed single drop; and (d) the morphology of a printed line. 

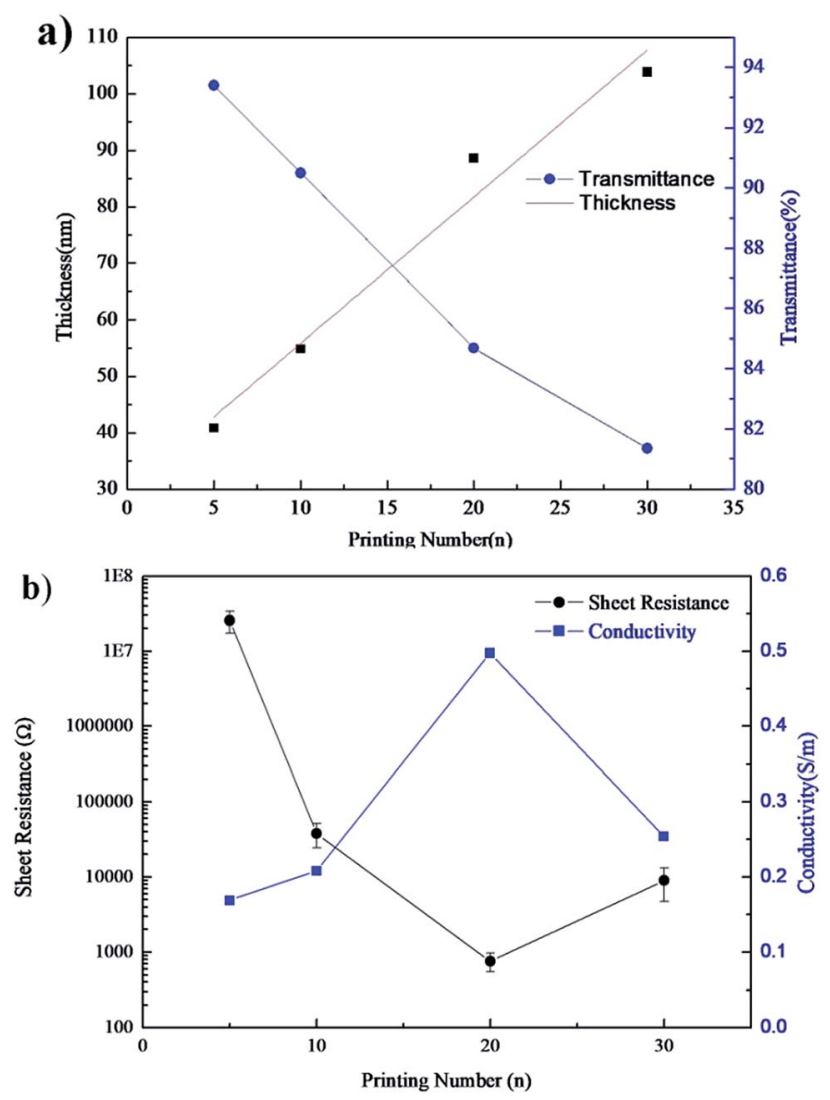

Fig. 4 Relationships between: (a) thickness and the transmittance and (b) the sheet resistance and the conductivity of the printed patterns and the printing numbers. than $1 \mu \mathrm{m}$ (Fig. 1a), and the thickness was around $1.8 \mathrm{~nm}$ (Fig. 1b), corresponding to the bilayer GO sheets. ${ }^{30}$

The substrate PET was put under the nozzle at a distance of $1 \mathrm{~mm}$, and its surface energy influenced the morphology and resolution of the printed patterns. ${ }^{31}$ To match the surface energy of the substrate with that of the ink, improving the wetting and spreading of the ink on PET, oxygen-plasma treatment was carried out to make PET hydrophilic. Comparison of the contact angles of the ink on the untreated (Fig. 2a) and treated substrates (Fig. 2b) showed that the contact angle decreased from $56.78^{\circ}$ to $8^{\circ}$, and the surface energy calculated from the Owens equation in two liquids ${ }^{32}$ increased from $42.52 \mathrm{~mJ} \mathrm{~m}^{-2}$ to $57.75 \mathrm{~mJ} \mathrm{~m}^{-2}$; this proved the wetting behavior of the ink on the substrate.

The prepared printable ink can remain stable for up to three months and have no aggregation, as shown in Fig. 3a. A droplet was injected from the nozzle successfully, separated into two drops, and finally merged into a single drop before exposing the treated PET (Fig. 3b); moreover, a satellite drop was not generated. There was almost no coffee ring observed in the printed single drop, as shown in Fig. 3c, and the line had flat edges and high resolution (Fig. 3d), with GO sheets distributed uniformly within its inner side.

\subsection{Morphology and conductivity of the printed patterns}

The number of printing passes directly influenced the thickness of the printed patterns. ${ }^{10}$ The thickness of the printed patterns scaled linearly with the printing times, as shown in Fig. 4. After printing 5 and 30 passes, the thickness of the printed patterns was $40.81 \mathrm{~nm}$ and $113.83 \mathrm{~nm}$, respectively, with each additional printing pass adding $5 \mathrm{~nm}$ thickness prior to IPL reduction. The
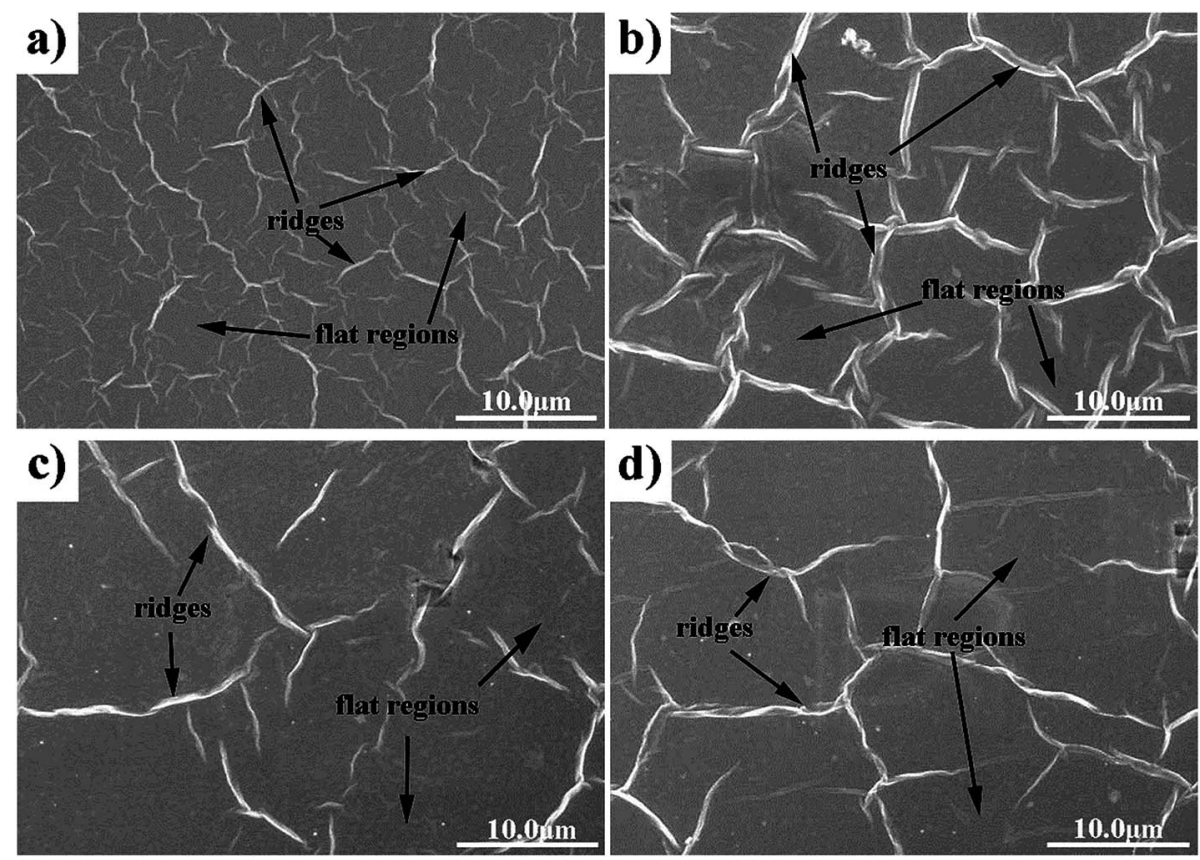

Fig. 5 SEM images of the films before IPL reduction with different printed passes on PET using GO ink: (a), (b), (c), and (d) corresponding to 5, 10, 20 , and 30 times, respectively. 
transmittance of the printed patterns decreased from $93.4 \%$ to $81.4 \%$ as the passes increased from 5 to 30 , respectively. As expected, the resistance decreased as the printing passes increased from 5 passes to 20 passes, corresponding to the conductivity increase, as shown in Fig. 4b. When 20 passes were printed, the resistance of the pattern was $56.77 \mathrm{M} \Omega$, and the conductivity of the pattern was $0.497 \mathrm{~S} \mathrm{~m}^{-1}$. It achieved the best conductivity among the experimentally printed patterns. The conductivity of the 30-pass printed pattern decreased as compared to that of the 20-pass printed pattern. This was
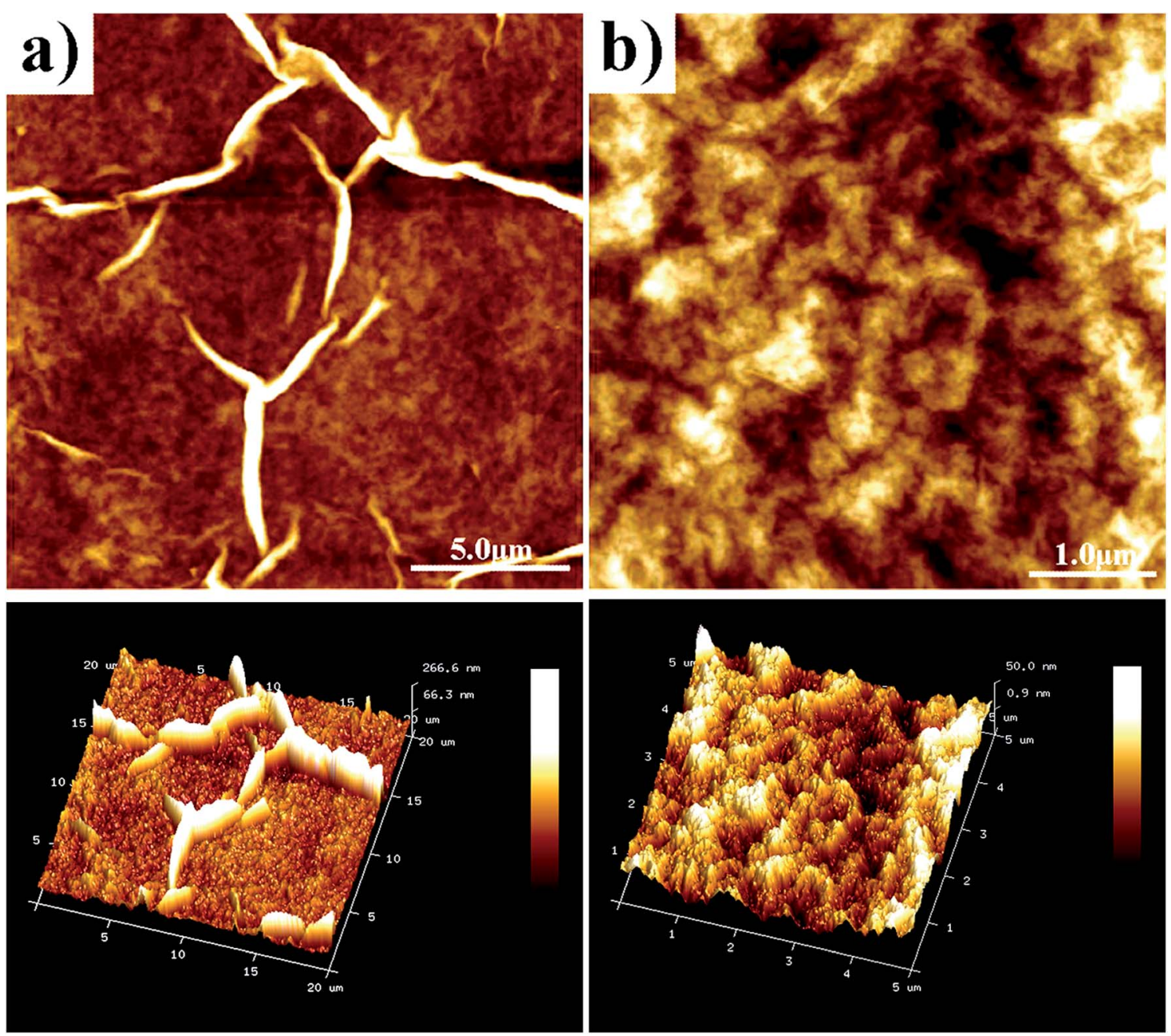

Fig. 6 AFM morphology and 3D profile images of the patterns printed using the GO ink (20 printing passes): (a) morphology of the ridges and (b) morphology of the flat regions.

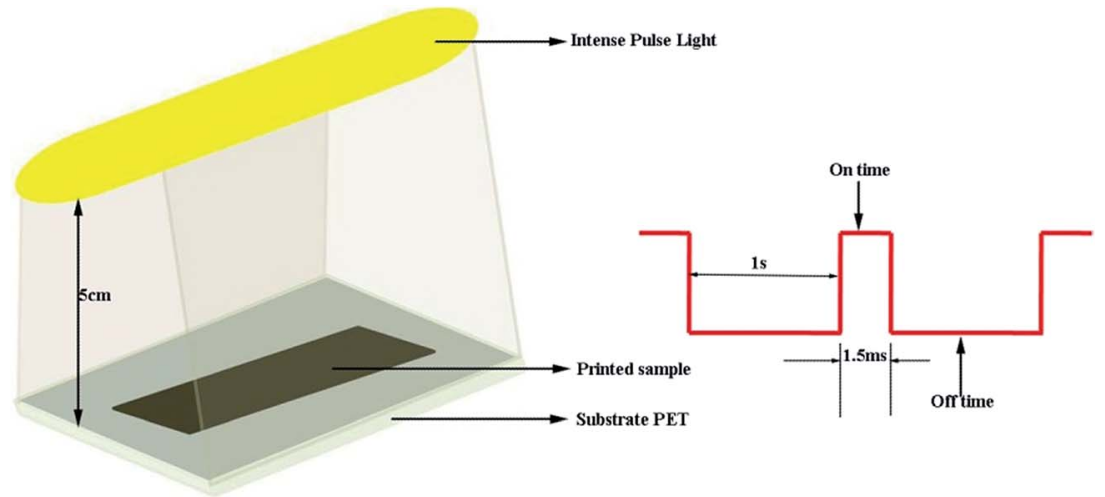

Fig. 7 Schematic of the IPL treatments. 
caused by more solvents in the patterns that affected the contact between GO sheets.

The morphology of the printed patterns is indicated in the SEM images (Fig. 5) and the AFM images (Fig. 6). Notably, there were many ridges and flat regions in the patterns (Fig. 5). Morphologies of the ridges and flat regions are shown in Fig. 6a and b, respectively. As shown in Fig. 6a, the height of the ridges was nearly $260 \mathrm{~nm}$, much larger than the thickness of GO as well as smaller than the lateral dimension of GO. As shown in Fig. $6 \mathrm{~b}$, the roughness of the flat region in the printed patterns was smaller than the roughness of the substrate PET; thus, it could be judged that there were still GO sheets on the flat basal plane. The ridges were produced because when GO inks were

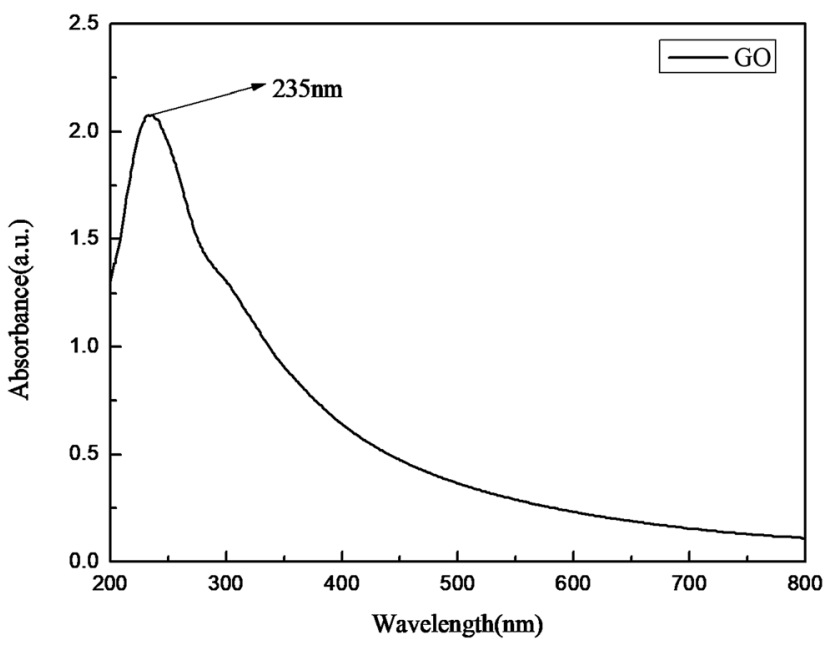

Fig. 8 Absorption spectrum of the GO sheets. exposed to the substrate, the solvents inside the patterns evaporated. During this process, GO sheets were carried with solvents and moved. When the moving GO sheets collided with each other, ridges were formed..$^{33,34}$

\subsection{Reduction of the printed patterns using IPL}

Reduction of the printed GO patterns was performed by photonic sintering. The xenon lamp emits a broad emission spectrum ranging from $190 \mathrm{~nm}$ to $800 \mathrm{~nm}$. Fig. 7 shows a schematic of the IPL reduction treatment under the atmospheric environment at room temperature. The distance between the xenon lamp and the samples was set at $5 \mathrm{~cm}$, and the patterns were treated under a single pulse for the time duration of $1.5 \mathrm{~ms}$.

Short-pulse bursts of high energy will generate a much higher heating rate and higher transient temperature. During the IPL irradiation, intensive light was delivered onto the printed GO films within a few milliseconds. The temperature of the films became high instantly because of the photo-thermal effect, which could break the bonds in the structure of GO sheets and lead to the reduction of the GO films. Moreover, the spectrum range of the xenon lamp matches well with that of the maximum resonance absorption peak of the GO sheets at $235 \mathrm{~nm}$, as shown in Fig. 8. As a result, the bonds in the GO sheets generated resonation with light in typical spectrum ranges; this accelerated the removal of oxygen functional groups and restoration of the original structure of graphene.

Compared with the patterns obtained before IPL, patterns treated with IPL exhibited fewer ridges, darker colors, and cracks (Fig. 9). The ridges became fewer and fewer as the number of printing passes increased. The number of cracks
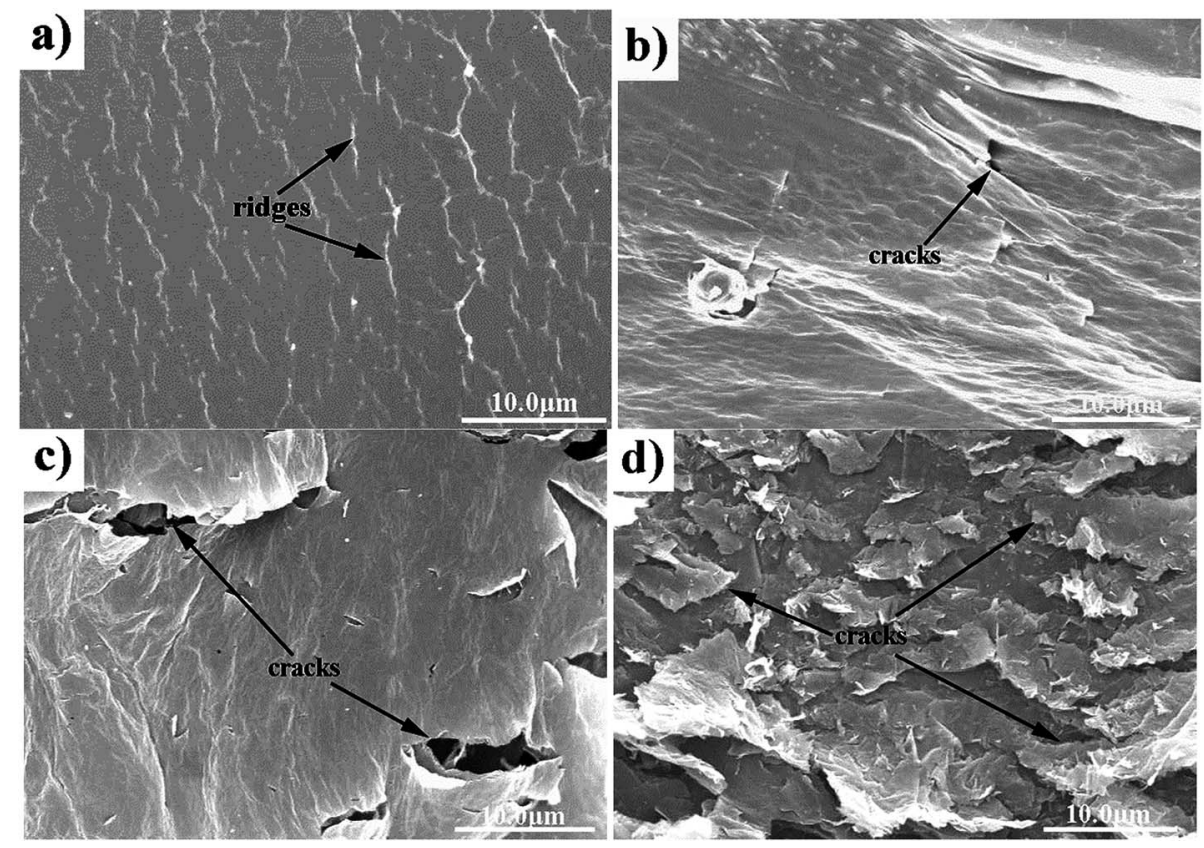

Fig. 9 SEM images of films after IPL reduction with different printed passes on PET using GO ink: (a), (b), (c), and (d) corresponding to 5, 10, 20, and 30 times, respectively. 
increased as the printing passes increased. After reduction, the patterns printed for 30 passes cracked completely and had weaker binding force with the substrate. It was seen that the conductivity of the patterns after reduction improved largely; especially, the resistance of the sample with 20 printing passes decreased to around $760.4 \Omega$, four orders of magnitude less than that of the patterns without reduction (Fig. 10). In their reported study, $\mathrm{ZhaO}^{30}$ et al. reduced thin GO films using $\mathrm{HI}$ acid, and the RGO films showed a sheet resistance of $840 \Omega$. Kong ${ }^{35}$ et al. developed a flexible electrode with a sheet resistance of $0.3 \mathrm{M} \Omega$ by reducing a GO electrode with an infrared heat lamp at a temperature of $200{ }^{\circ} \mathrm{C}$. Compared with the abovementioned reports, the 20 printing-passes pattern after IPL treatment exhibited superior conductivity in this study. When the printed patterns were placed under light, GO sheets were reduced by the instantaneous high temperature, and the solvent evaporated rapidly. Thus, the conductivity of the patterns after IPL improved. On the other hand, solvents tended to escape from regions with ridges. This caused cracks and reduced the number of ridges on the pattern surface. Although there were more cracks on the pattern surface, the resistance of the pattern with 20 passes after IPL treatment was lower than that of the patterns with 5 and 10 passes. It was mainly because more reduced GO sheets in the pattern with 20 passes resulted in more inter-flake junctions between reduced GO sheets as the printing passes increased. It led to the formation of more conduction pathways for electrons to immigrate and the improvement of the pattern resistance. Moreover, the volume of solvents inside the patterns increased with the increasing printing passes. It caused more solvent escaping, and thus, more cracks occurred. Therefore, compared with that of the pattern with 20 passes, the higher resistance of the 30 printingpasses pattern was attributed to too many cracks on its surface that seriously impeded the reduced GO sheets contact.

To confirm the effectiveness of the GO reduction by IPL, XPS spectra of the GO powder and printed samples before and after reduction were compared. The XPS C 1s spectra for all samples indicated typical characteristics of graphene materials. The

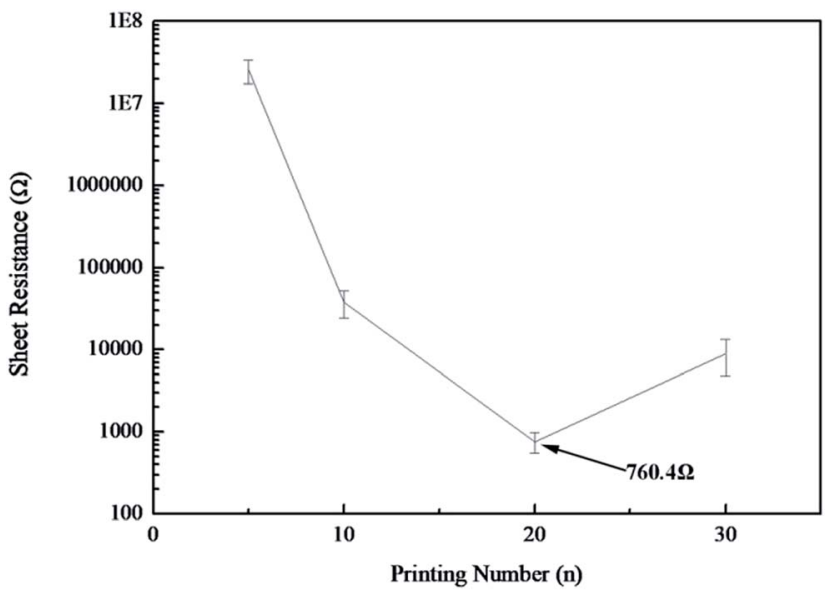

Fig. 10 Relationship between the printing numbers and the resistance after IPL reduction. dominant peak around $284.5 \mathrm{eV}$ was ascribed to the region of $\mathrm{C}=\mathrm{C}$, the peak around $285.3 \mathrm{eV}$ was ascribed to the region of $\mathrm{C}-\mathrm{C}$, the peak between $286.5 \mathrm{eV}$ and $287.5 \mathrm{eV}$ is within the carbon region of $\mathrm{C}-\mathrm{O}$ and $\mathrm{C}=\mathrm{O}$, and the peak around $289.0 \mathrm{eV}$ is the signal of carboxyl carbon. ${ }^{29,36}$ Interestingly, it was seen that as compared to that in the GO powder (Fig. 11a), the percentage of $\mathrm{C}-\mathrm{C}$ bonding and $\mathrm{C}-\mathrm{O}$ bonding in the printed patterns before reduction became larger, respectively increasing from $30.18 \%$ and $38.33 \%$ to $34.01 \%$ and $40.91 \%$, due to carbon-
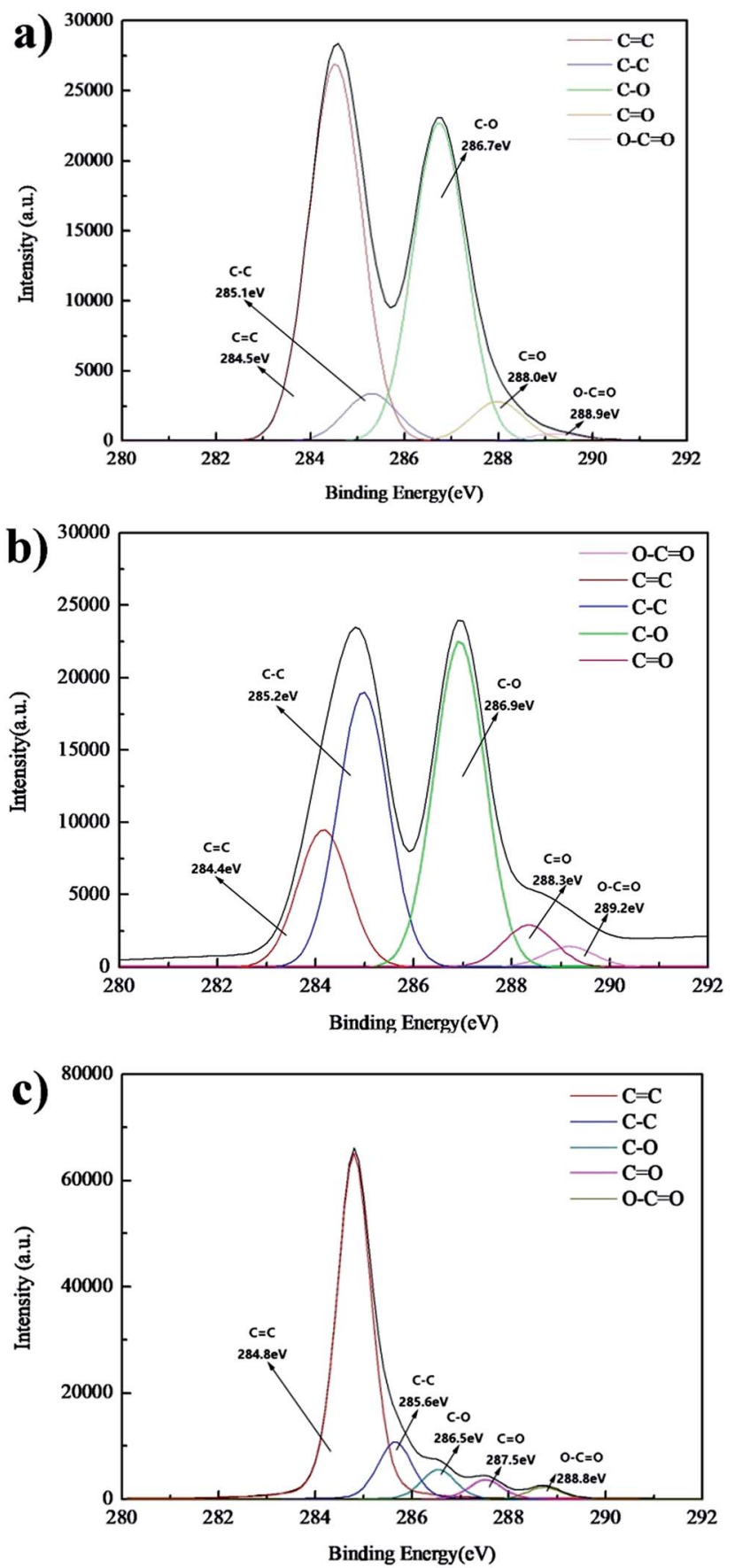

Fig. 11 XPS spectra of (a) GO powders; (b) GO printed patterns (20 passes) before IPL; (c) GO printed patterns (20 passes) after IPL. 
oxygen bonding of the solvents. Compared with the printed patterns before reduction, the percentage of $\mathrm{C}=\mathrm{C} / \mathrm{C}-\mathrm{C}$ bonding in patterns after IPL was significantly enhanced from the

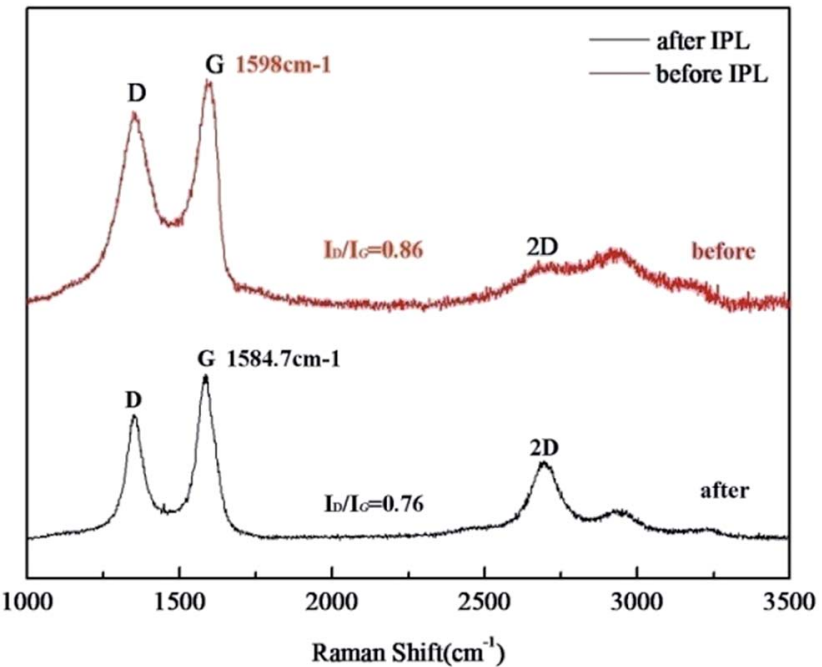

Fig. 12 Raman spectra of the patterns (20 passes) before and after IPL reduction.
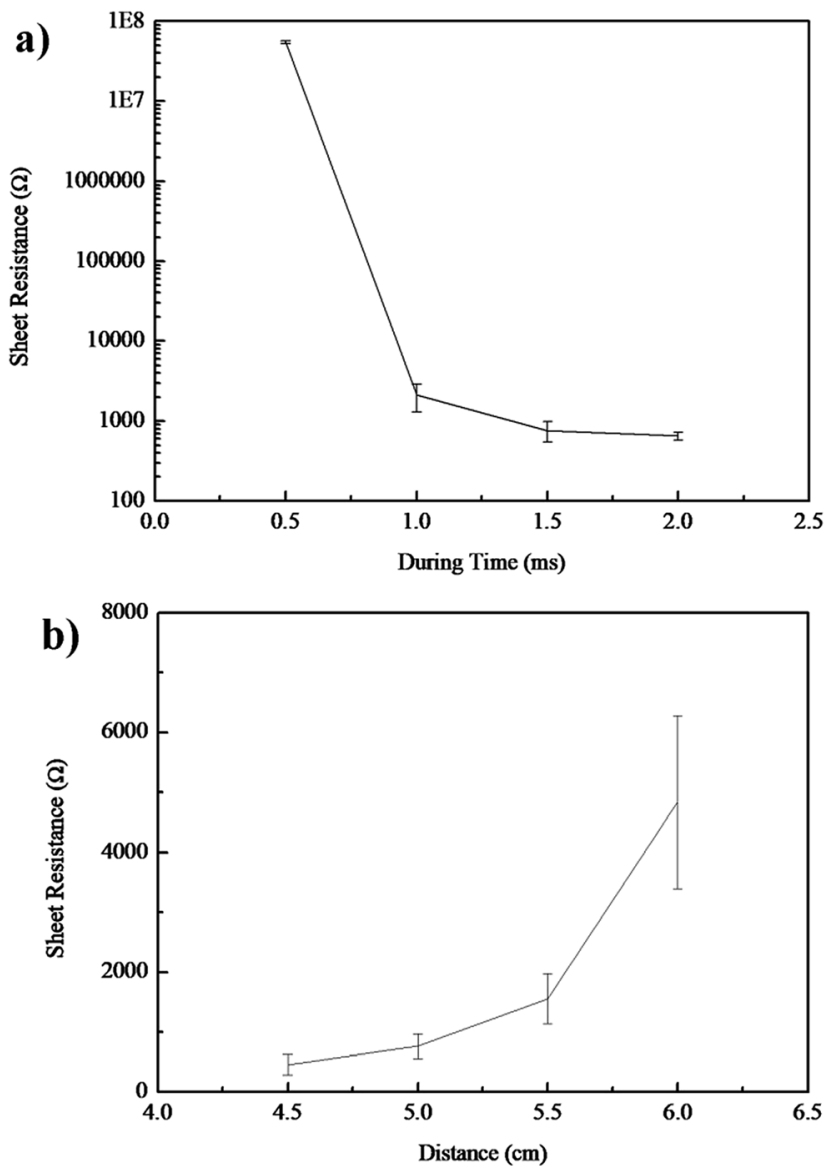

Fig. 13 Relationship between resistance and (a) during time of the pulse; (b) distance between patterns and light. original $50.66 \%$ to $86.88 \%$, even higher than that of the GO powder $(54.30 \%)$. Moreover, the percentage of $\mathrm{C}-\mathrm{O}$ decreased from $40.91 \%$ for the patterns before reduction to $6.41 \%$ after reduction. It proved that the $\pi$-conjugated structure of graphene was restored, and the solvents in the samples evaporated; this increased the electrical conductivity of the patterns after IPL. ${ }^{37,38}$ In the Raman spectra (Fig. 12), patterns after IPL had a lower ratio for the intensity of the $D$ band to that of the $G$ band $\left(I_{\mathrm{D}} / I_{\mathrm{G}}\right)$ than patterns before IPL, and the $\mathrm{G}$ band in the patterns after IPL downshifted from $1598 \mathrm{~cm}^{-1}$ for the patterns before IPL to around $1584.7 \mathrm{~cm}^{-1}$; this indicated that the conjugated structure was restored, and the defect density in the carbon basal plane was lower, closer to that of pristine graphene. ${ }^{39,40}$

To explore optimized reduction parameters, the distance between the light and the samples and time duration of the pulses were changed, and their effects on the conductivity and morphology of the patterns were investigated..$^{28}$ As shown in Fig. 13, resistance of the patterns after IPL decreased with the increasing pulse duration time or decreasing distance, and it achieved the highest value when the distance was increased to $4.5 \mathrm{~cm}$ or the duration time was up to $2.0 \mathrm{~ms}$. However, binding force between the patterns and the substrate was too low to keep the patterns adhered to the substrate; thus, the optimized parameters were set as $1.5 \mathrm{~ms}$ and $5.5 \mathrm{~cm}$.

\subsection{Flexibility of the reduced patterns}

Resistances of the printed patterns on PET under different bending cycles were measured (Fig. 14) to reveal the flexibility of the printed patterns after IPL. When the 20 passes-printed patterns were bent outwards by $180^{\circ}$ after 1000 bending cycles, there were no observable cracks on the plane, and the patterns became levelled again. The change of resistance was still acceptable. This result indicates that inkjet printing of the GO ink can generate flexible patterns that satisfy the requirements of flexible devices.

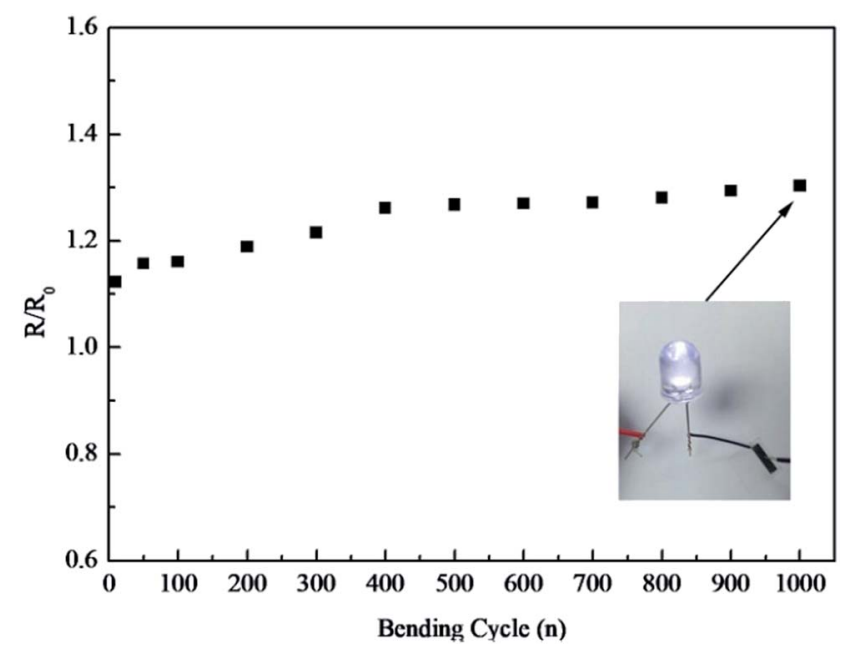

Fig. 14 Resistance measured as a function of bending cycles for GO patterns on PET after IPL reduction. 


\section{Conclusions}

In summary, a printable GO ink was successfully prepared in solvents involving deionized water, ethylene glycol, and ethanol. Patterns with a high resolution were inkjet-printed with the prepared inks. Printed GO patterns were reduced rapidly with IPL treatment to achieve low resistance and fine morphology. Upon printing 20 passes, the resistance achieved was as low as $760.4 \Omega$ after IPL reduction. Compared with the resistance of the patterns before IPL treatment, the resistance of the patterns after IPL was lowered by nearly four orders of magnitude. Moreover, after bending outwards $180^{\circ}$ for 1000 cycles, the resistance of the reduced GO patterns changed slightly, and this was still acceptable. Furthermore, the substrate PET, sensitive to high temperatures, was unbroken after IPL. This establishes that GO ink can be reduced by the IPL method, and this result can be used as a reference for flexible, printed electronics such as sensors and electrodes.

\section{Conflicts of interest}

There are no conflicts to declare.

\section{References}

1 T. Felice, H. Tawfique, W. Weiping, S. Zhipei, L. Antonio, S. K. Tero, H. Gen-Wen, J. Sungjune, B. Francesco, J. P. Philip, C. Daping and C. F. Andrea, ACS Nano, 2012, 6(4), 2992-3006.

2 M. H. Ervin, L. T. Le and W. Y. Lee, Electrochim. Acta, 2014, 147, 610-616.

3 J. Viventi, D. H. Kim, L. Vigeland, E. S. Frechette, J. A. Blanco, Y. S. Kim, A. E. Avrin, V. R. Tiruvadi, S. W. Hwang, A. C. Vanleer, D. F. Wulsin, K. Davis, C. E. Gelber, L. Palmer, J. Van der Spiegel, J. Wu, J. Xiao, Y. Huang, D. Contreras, J. A. Rogers and B. Litt, Nat. Neurosci., 2011, 14, 1599-1605.

4 D. Tobjork and R. Osterbacka, Adv. Mater., 2011, 23, 19351961.

5 L. F. Lima, C. F. Matos, L. C. Gonçalves, R. V. Salvatierra, C. E. Cava, A. J. G. Zarbin and L. S. Roman, J. Phys. D: Appl. Phys., 2016, 49, 105106.

6 T. Vuorinen, M. Zakrzewski, S. Rajala, D. Lupo, J. Vanhala, K. Palovuori and S. Tuukkanen, Adv. Funct. Mater., 2014, 24, 6340-6347.

7 X. Huang, T. Leng, M. Zhu, X. Zhang, J. Chen, K. Chang, M. Aqeeli, A. K. Geim, K. S. Novoselov and Z. Hu, Sci. Rep., 2015, 5, 18298.

8 S. Wünscher, R. Abbel, J. Perelaer and U. S. Schubert, J. Mater. Chem. C, 2014, 2, 10232-10261.

9 H. Zhang, A. Xie, Y. Shen, L. Qiu and X. Tian, Phys. Chem. Chem. Phys., 2012, 14, 12757-12763.

10 K. Y. Shin, J. Y. Hong and J. Jang, Adv. Mater., 2011, 23, 21132118.

11 B. Zhan, C. Li, J. Yang, G. Jenkins, W. Huang and X. Dong, Small, 2014, 10, 4042-4065.
12 W. Shen, X. Zhang, Q. Huang, Q. Xu and W. Song, Nanoscale, 2014, 6, 1622-1628.

13 Y. Gao, W. Shi, W. Wang, Y. Leng and Y. Zhao, Ind. Eng. Chem. Res., 2014, 53, 16777-16784.

14 E. B. Secor, P. L. Prabhumirashi, K. Puntambekar, M. L. Geier and M. C. Hersam, J. Phys. Chem. Lett., 2013, 4, 1347-1351.

15 J. Li, F. Ye, S. Vaziri, M. Muhammed, M. C. Lemme and M. Ostling, Adv. Mater., 2013, 25, 3985-3992.

16 V. Dua, S. P. Surwade, S. Ammu, S. R. Agnihotra, S. Jain, K. E. Roberts, S. Park, R. S. Ruoff and S. K. Manohar, Angew. Chem., 2010, 49, 2154-2157.

17 K. S. Novoselov, V. I. Fal'ko, L. Colombo, P. R. Gellert, M. G. Schwab and K. Kim, Nature, 2012, 490, 192-200.

18 C.-L. Lee, C.-H. Chen and C.-W. Chen, Chem. Eng. J., 2013, 230, 296-302.

19 T. Le, V. Lakafosis, T. Thai, Z. Y. Lin and M. Tentzeris, 2012 International Conference on Electromagnetics In Advanced Applications (Iceaa), 2012, pp. 954-957.

20 Y. Su, S. Jia, J. Du, J. Yuan, C. Liu, W. Ren and H. Cheng, Nano Res., 2015, 8, 3954-3962.

21 L. Huang, Y. Huang, J. Liang, X. Wan and Y. Chen, Nano Res., 2011, 4, 675-684.

22 A. Kamyshny and S. Magdassi, Small, 2014, 10, 35153535.

23 L. T. Le, M. H. Ervin, H. Qiu, B. E. Fuchs and W. Y. Lee, Electrochem. Commun., 2011, 13, 355-358.

24 K. Chi, Z. Zhang, J. Xi, Y. Huang, F. Xiao, S. Wang and Y. Liu, ACS Appl. Mater. Interfaces, 2014, 6, 1631216319.

25 F. Xiao, S. Yang, Z. Zhang, H. Liu, J. Xiao, L. Wan, J. Luo, S. Wang and Y. Liu, Sci. Rep., 2015, 5, 9359.

26 S. Das, D. Cormier and S. Williams, Procedia Manufacturing, 2015, 1, 366-377.

27 J. S. Kang, J. Ryu, H. S. Kim and H. T. Hahn, J. Electron. Mater., 2011, 40, 2268-2277.

28 E. B. Secor, B. Y. Ahn, T. Z. Gao, J. A. Lewis and M. C. Hersam, Adv. Mater., 2015, 27, 6683-6688.

29 B. Derby, Annu. Rev. Mater. Res., 2010, 40, 395-414.

30 J. P. Zhao, S. F. Pei, W. C. Ren, L. B. Gao and H. M. Cheng, ACS Nano, 2010, 4, 5245-5252.

31 S. Santra, G. Hu, R. C. Howe, A. De Luca, S. Z. Ali, F. Udrea, J. W. Gardner, S. K. Ray, P. K. Guha and T. Hasan, Sci. Rep., 2015, 5, 17374.

32 H. Tavana, C. N. Lam, K. Grundke, P. Friedel, D. Y. Kwok, M. L. Hair and A. W. Neumann, J. Colloid Interface Sci., 2004, 279, 493-502.

33 M. Kuang, L. Wang and Y. Song, Adv. Mater., 2014, 26, 69506958.

34 H. Kim, J. I. Jang, H. H. Kim, G. W. Lee, J. A. Lim, J. T. Han and K. Cho, ACS Appl. Mater. Interfaces, 2016, 8, 31933199.

35 D. Kong, L. T. Le, Y. Li, J. L. Zunino and W. Lee, Langmuir, 2012, 28, 13467-13472.

36 K. Y. Shin, J. Y. Hong and J. Jang, Chem. Commun., 2011, 47, 8527-8529. 
37 M. Bai, J. Chen, W. Wu, X. Zeng, J. Wang and H. Zou, Colloids Surf., A, 2016, 490, 59-66.

38 Y. Su, J. Du, D. Sun, C. Liu and H. Cheng, Nano Res., 2013, 6, 842-852.
39 H. A. Becerril, J. Mao, Z. Liu, R. M. Stoltenberg, Z. Bao and Y. Chen, ACS Nano, 2008, 2, 463-470.

40 A. C. Ferrari and D. M. Basko, Nat. Nanotechnol., 2013, 8, 235-246. 